\title{
Cabazitaxel operates anti-metastatic and cytotoxic via apoptosis induction and stalls brain tumor angiogenesis
}

\author{
Ali Ghoochani ${ }^{1}$, Gökce Hatipoglu Majernik ${ }^{1,2}$, Tina Sehm ${ }^{1}$, Sven Wach ${ }^{3}$, Michael \\ Buchfelder $^{1}$, Helge Taubert ${ }^{3}$, Ilker Y. Eyupoglu ${ }^{1, *}$, Nicolai Savaskan ${ }^{1,4, *}$ \\ ${ }^{1}$ Translational Cell Biology \& Neurooncology Laboratory, Department of Neurosurgery, Universitätsklinikum Erlangen, Medical \\ School of The Friedrich-Alexander University (FAU) of Erlangen - Nürnberg, Erlangen, Germany \\ ${ }^{2}$ Present Address: Department of Neurosurgery, Medizinische Hochschule Hannover (MHH), Hannover, Germany \\ ${ }^{3}$ Department of Urology, Universitätsklinikum Erlangen, Medical School of The Friedrich-Alexander University (FAU) of \\ Erlangen - Nürnberg, Erlangen, Germany \\ ${ }^{4}$ BiMECON Ent., Berlin, Germany \\ *These authors have contributed equally to this work
}

Correspondence to: Nic Savaskan, email: savaskan@gmx.net; nic.savaskan@gmail.com

Keywords: gliomas, neurotoxicity, taxane, angiogenesis, cell death

Received: February 1, $2016 \quad$ Accepted: April 28, $2016 \quad$ Published: May 18, 2016

\section{ABSTRACT}

Taxanes target microtubules and are clinically established chemotherapeutic agents with proven efficacy in human cancers. Cabazitaxel (XRP-6258, Jevtana ${ }^{\circledR}$ ) is a second generation semisynthetic taxane with high chemotherapeutic potential in prostate cancer. There, cabazitaxel can overcome docetaxel-resistant prostate cancer. Here, we tested the effects of cabazitaxel on glioma cells, and non-transformed cells such as neurons and astrocytes. Cabazitaxel operates highly toxic in various human glioma cells at nanomolar concentrations. In contrast, primary astrocytes and neurons are not affected by this agent. Cabazitaxel disrupts cytoskeletal F-actin fibers and induces apoptotic cell death in gliomas. Moreover, cabazitaxel displayed highest efficacy in inhibiting glioma cell migration and invasion. Here we demonstrate that cabazitaxel inhibited tumor migration already at $1 \mathrm{nM}$. We also tested cabazitaxel in the ex vivo VOGiM assay. Cabazitaxel stalled glioma growth and at the same time inhibited tumor-induced angiogenesis. In summary, we found that cabazitaxel operates as an apoptosis-inducing gliomatoxic agent with strongest effects on migration and invasive growth. Thus, our report uncovered cabazitaxel actions on gliomas and on the brain tumor microenvironment. These data reveal novel aspects for adjuvant approaches when applied to brain tumor patients.

\section{INTRODUCTION}

Malignant primary brain tumors are the most common type of solid tumors in the CNS affecting children and adults. So far, the prognosis for patients diagnosed with central nervous system tumors such as glioblastomas (GBM) stagnated in recent years, largely due to missing novel efficient and at the same time safe chemotherapeutic agents [1]. The poor prognosis is often ascribed to incomplete tumor resection and the resistance and non-responsiveness of gliomas to current therapeutics. In addition, treatment of these tumors often results in significant long-term disabilities and reduction in quality of life $[2,3]$. Therefore, finding new therapies with higher efficacy and at the same time less neurotoxic side effects is essential for improving event-free and overall survival in GBM patients.

During recent years the applicability of drugs or small molecule inhibitors on glioma cells particularly on vascularization and the tumor-immune interaction has been becoming center of interest [4-6]. However, one obstacle in delivering therapeutics to tumors of the central nervous system is tumor-induced angiogenesis which challenges the blood-brain barrier and absorption rate (BBB) [4, 7-9]. The capillary endothelial cells of brain tumors with tight junctions' trait are different and distinguishable from endothelial cells of other tissues in that they lack penetration. This feature has clinical 
implications in that therapeutic agents are impeded to passively diffuse into the brain $[10,11]$. Therefore, discovering small molecule drugs passing the blood-brain barrier more efficient would challenge efficacy in targeting tumor growth rate and pathological tumor angiogenesis.

Cabazitaxel is a second-generation semisynthetic taxane which is currently Food and Drug Agency (FDA) and European Medicines Agency (EMA) approved for treatment of castration-resistant prostate cancer [12]. Indeed, the applicability of first generation taxane therapies (paclitaxel and docetaxel) displayed some limitations. Paclitaxel and docetaxel are high-affinity substrates for ATP-dependent multidrug-resistant pumps $[13,14]$. Tumor-induced activation of multidrugresistant pumps leads to lower penetration and effective concentrations of therapeutic agents across the $\mathrm{BBB}$ due to challenged endothelial influx/efflux ratios [15]. In contrast to paclitaxel and docetaxel, the affinity of multidrugresistant pumps for cabazitaxel is much lower, indicating a better efficacy of cabazitaxel in brain tumors. Moreover, in vivo evidence for the distribution of cabazitaxel throughout the brain and the capacity of the substance to get absorbed by endothelial cells of the BBB has recently been shown [16].

Therefore, in this study we tested whether cabazitaxel treatment can successfully fight primary brain tumor growth and whether cabazitaxel can efficiently reverse tumor angiogenesis. In this study we used the ex vivo vascular glioma impact method (VOGiM) to investigate the influence of gliomas and chemotherapeutics on the tumor microenvironment and angiogenesis [17]. Our results suggest that application of cabazitaxel does not only prevent glioma growth but also induce enhanced tumor cell death compared to non-tumoral area. Moreover, we show that cabazitaxel treatment reduces tumor-induced angiogenesis while normal non-transformed brain cells and endothelial cells are not affected by this agent.

\section{RESULTS}

\section{Cabazitaxel reduces glioma cell growth and survival}

To study the effects of cabazitaxel on brain cancer cell proliferation and survival, we used two human glioma cell lines (T98G and U87) which were treated with a wide range of cabazitaxel concentrations. Glioma cells were seeded in number of $3 \times 10^{3}$ cells in 96-wells plates for a day prior drug application. Next day we treated cells with cabazitaxel for three days at concentrations of 1 to $100 \mathrm{nM}$ in order to investigate its glioma toxicity potential (Figure 1). In T98G and U87 glioma cell lines, cabazitaxel treatment significantly reduced cell survival and proliferation. We found that a concentration of 2.5 nM cabazitaxel was sufficient to inhibit cell proliferation (Figure 1A, 1B). However, $1 \mathrm{nM}$ cabazitaxel was also effective to induce $20 \%$ cell death effect on T98G cells (Figure 1A). Taken together, these results demonstrate that cabazitaxel is effective in reducing glioma proliferation although the impact stagnates at $60 \%$ even at higher concentrations.
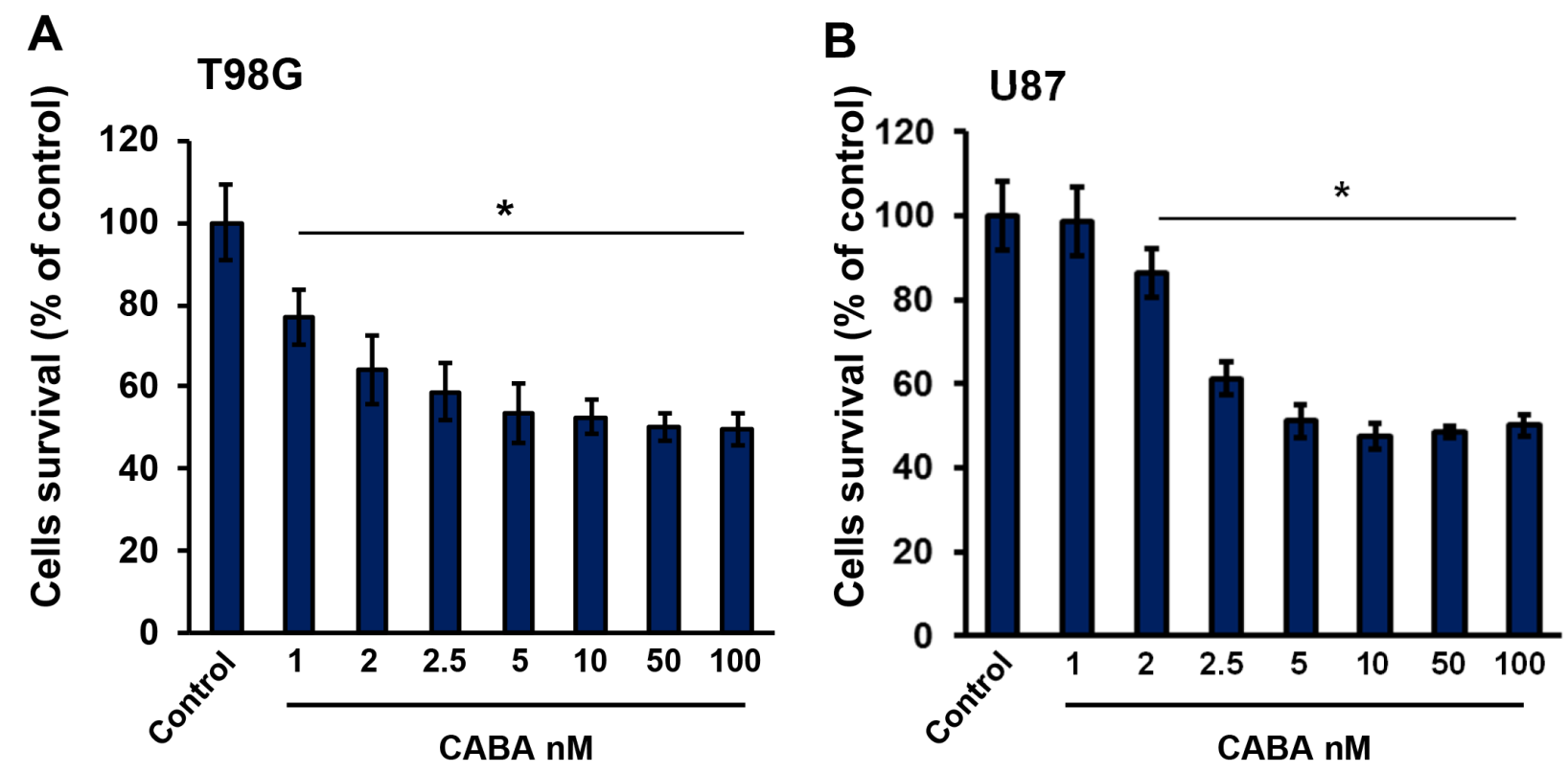

Figure 1: Cell proliferation and survival under cabazitaxel at different concentrations. A. T98G and B. U87 cell lines were treated with 1, 2, 2.5, 5, 10, 50 and $100 \mathrm{nM}$ cabazitaxel for 3 days. MTT assay was implicated to measure cell survival as described in material and methods. Experiment was performed in three independent repetitions. Statistical analysis was performed with One-way $\operatorname{ANOVA}(* \mathrm{P}<0.05$, mean is given \pm s.e.m. $)$. 


\section{Cabazitaxel is not toxic to primary neurons and astrocytes}

In a next step, we isolated rat hippocampal neurons and astrocytes and evaluated whether cabazitaxel impacts selectively on gliomas or is a general toxic agent even for non-transformed brain cells. Therefore, we treated isolated hippocampal neurons and astrocytes with a range of 1 to $10 \mathrm{nM}$ cabazitaxel which appeared to be effective on glioma cells (Figure 1). Cabazitaxel treatment did not adversely change neuronal or astrocyte branches at various concentrations compared to untreated controls (Figure $2 \mathrm{~A})$. Both neurons and astrocytes displayed a preserved quality in morphology, branches and expression of Tuj-1 and GFAP neuronal and astrocyte markers, respectively, (Figure 2A) during five days of treatment. All tested concentrations did not significantly challenged both neuronal and astroglial marker expression (Figure 2B). Therefore, these results confirm cabazitaxel as a selective toxic agent for glioma cells which is not toxic for resident brain cells.

\section{Cabazitaxel induces apoptotic cell death in gliomas}

Hence, we investigated the mechanisms of cabazitaxel-induced cell death in gliomas. For this we facilitated human glioma cells and applied effective nanomolar levels of cabazitaxel (Figure 3). Cell cycle profiling of glioma cells following cabazitaxel treatment revealed a left shift of cabazitaxel treated cells towards the sub- $\mathrm{G}_{1}$ phase indicating increased apoptotic or

\section{A}
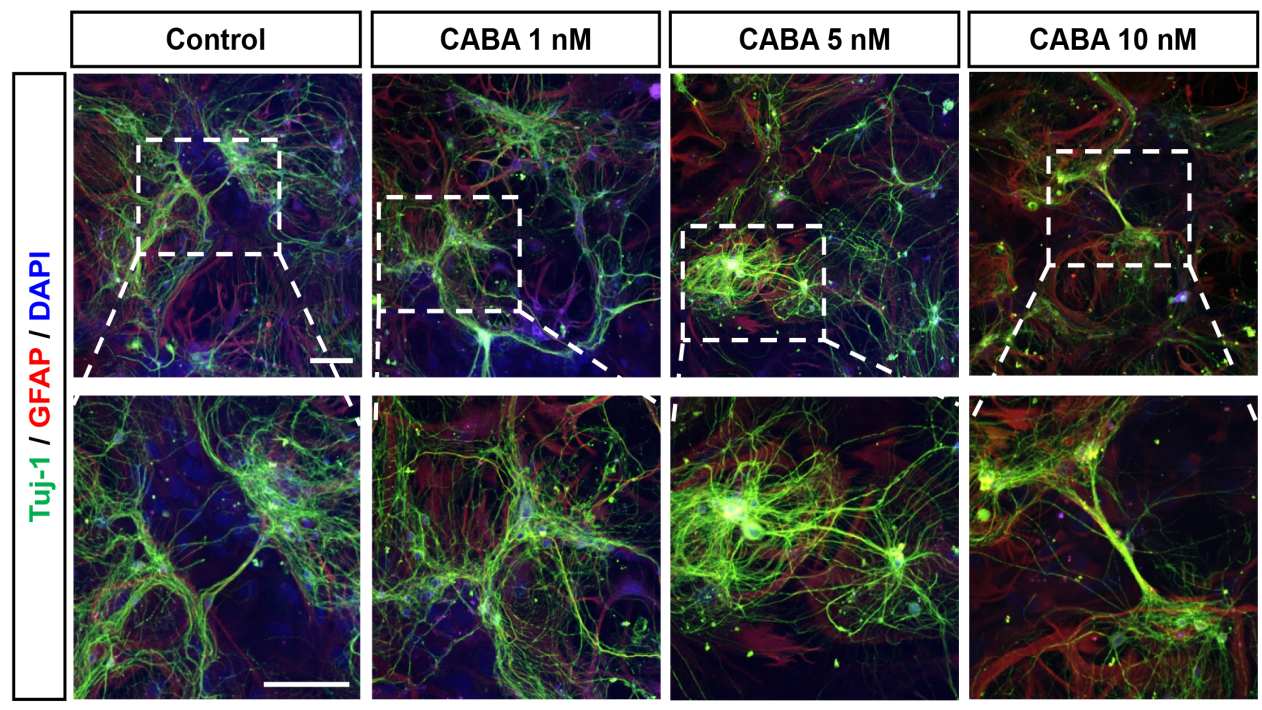

B
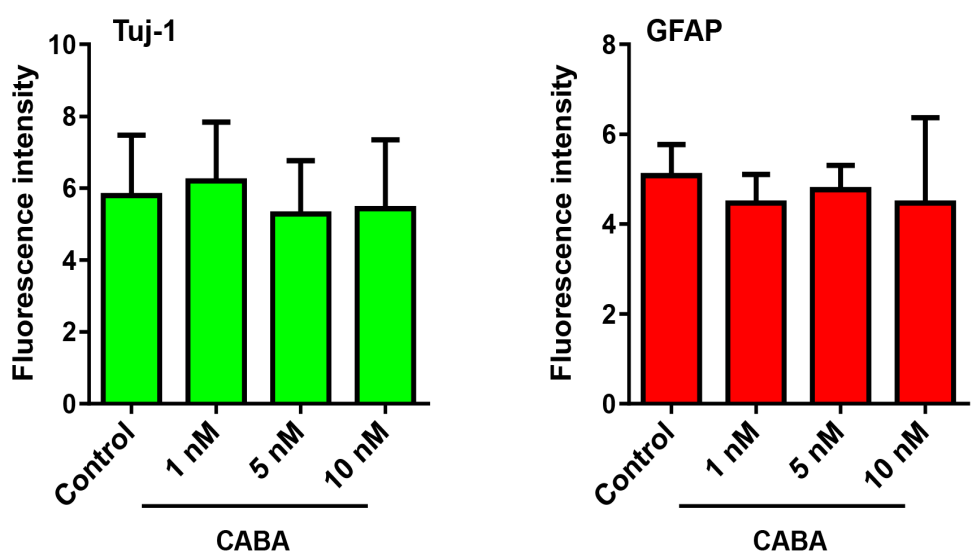

Figure 2: Cabazitaxel is not toxic to primary neurons and astrocytes. A. Isolated primary hippocampal neurons and astrocytes were treated with 1,5 and $10 \mathrm{nM}$ cabazitaxel for 3 days. Neurons and astrocyte were stained with anti Tuj-1 (green) and GFAP (red) respectively. Scale bar represents $100 \mu \mathrm{m}$. B. Quantification of Tuj-1 ( $\beta$-III tubulin) and GFAP immunostaining $(n=3)$. Statistical analysis was performed with One-way ANOVA (mean is given \pm s.e.m.). 
necrotic cell death (Figure 3A). Also, cabazitaxel induced a significant right shift to the $G_{2}$ phase which is equivalent to a growth arrest response (Figure 3A, 3B). We further performed fluorescence activated cell sorting-based analysis to uncover the mode of cabazitaxel-induced cell

A

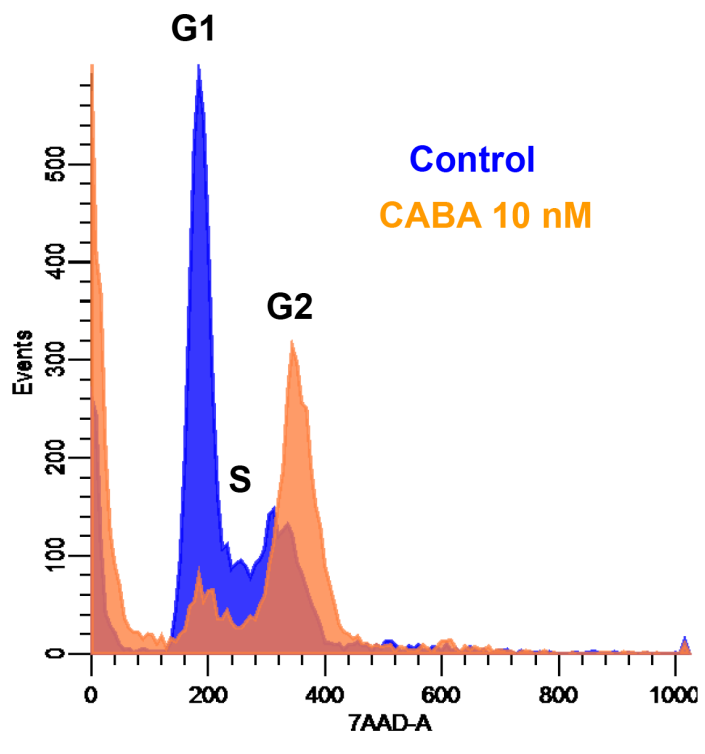

C

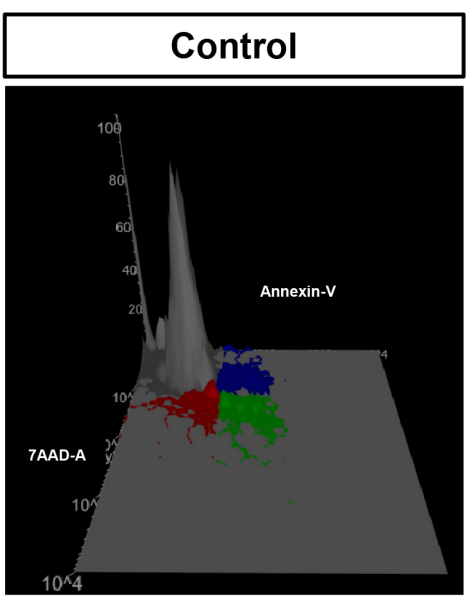

B

death. Apoptosis was monitored via Annexin V staining and 7AAD staining was used as a marker for the cell death end stage (Figure 3C, 3D). We quantified apoptotic and necrotic cell death and found that cabazitaxel induces mainly apoptotic cell death (Figure 3C, 3D).

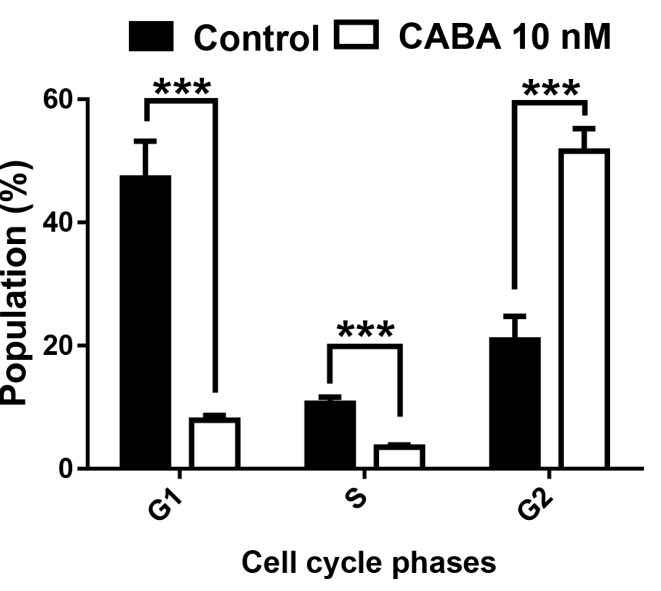

D

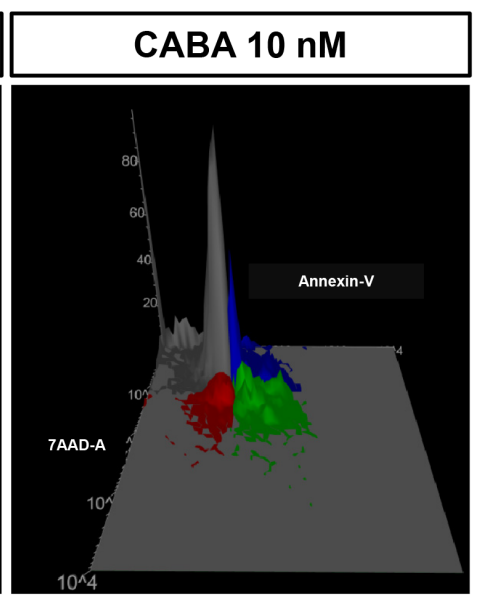

7AAD

Annexin V

7AAD+Annexin V

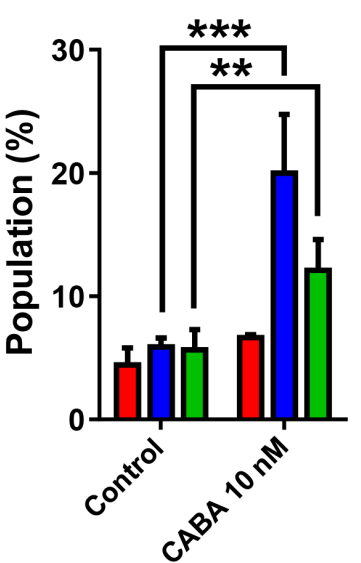

Figure 3: Cabazitaxel induces apoptotic cell death in gliomas. A. Cell cycle profiling of glioma cells following cabazitaxel (CABA) treatment. Control cell population is given in blue, cabazitaxel treated cell population is given in orange. Note the left shift of CABA treated cells towards the sub- $G_{1}$ phase and the right shift to the $G_{2}$ phase. B. Quantification of the cell cycle analysis in human glioma cells. Differences were considered statistically significant with values given as mean \pm s.e.m. $(n=3$ independent experiments per group; unpaired t-test, $* * * \mathrm{P}<0.001)$. C. Fluorescence activated cell sorting-based analysis for apoptosis following cabazitaxel application. Apoptosis was monitored via Annexin V staining given in blue (early apoptosis and membrane integrity) and Annexin V/7AAD double staining given in green (late apoptosis, cell death end stage). The 7AAD pool is shown in red. D. Quantification of various apoptotic and cell death fractions. Differences were considered statistically significant with values given as mean \pm s.e.m. $(n=3$ independent experiments per group; unpaired t-test, $* * \mathrm{P}<0.005 * * * \mathrm{P}<0.001)$. 


\section{Cabazitaxel suppresses glioma cell migration}

As we could demonstrate that cabazitaxel adversely affects glioma cell survival and proliferation, the question raised whether cabazitaxel is also able to reduce glioma cell migration. Therefore, we performed an assessment for in vitro glioma cell migration under cabazitaxel treatment. For this, glioma cells were cultured and allowed to reach more than $90 \%$ confluency. Afterwards, monolayered glioma cells were scratched to create a distinct and quantifiable cell-free area. Along with the scratch, glioma cells were treated with different concentration
1,5 and $10 \mathrm{nM}$ of cabazitaxel to measure the twodimensional movement of untreated control samples and cabazitaxel treated glioma cells at different time point 0 , 12 and 24 hours after scratch (Figure 4). As representative images illustrate, increasing concentration of cabazitaxel significantly inhibits migration and motility of glioma cells in comparison to untreated controls (Figure 4). The distance of scratch borders measured $86 \mu \mathrm{m}$ after 24 hours in untreated control samples while this distance was 264 , 370 and $430 \mu \mathrm{m}$ wide after 15 and $10 \mathrm{nM}$ cabazitaxel treatment, respectively (Figure 4A). These results
A
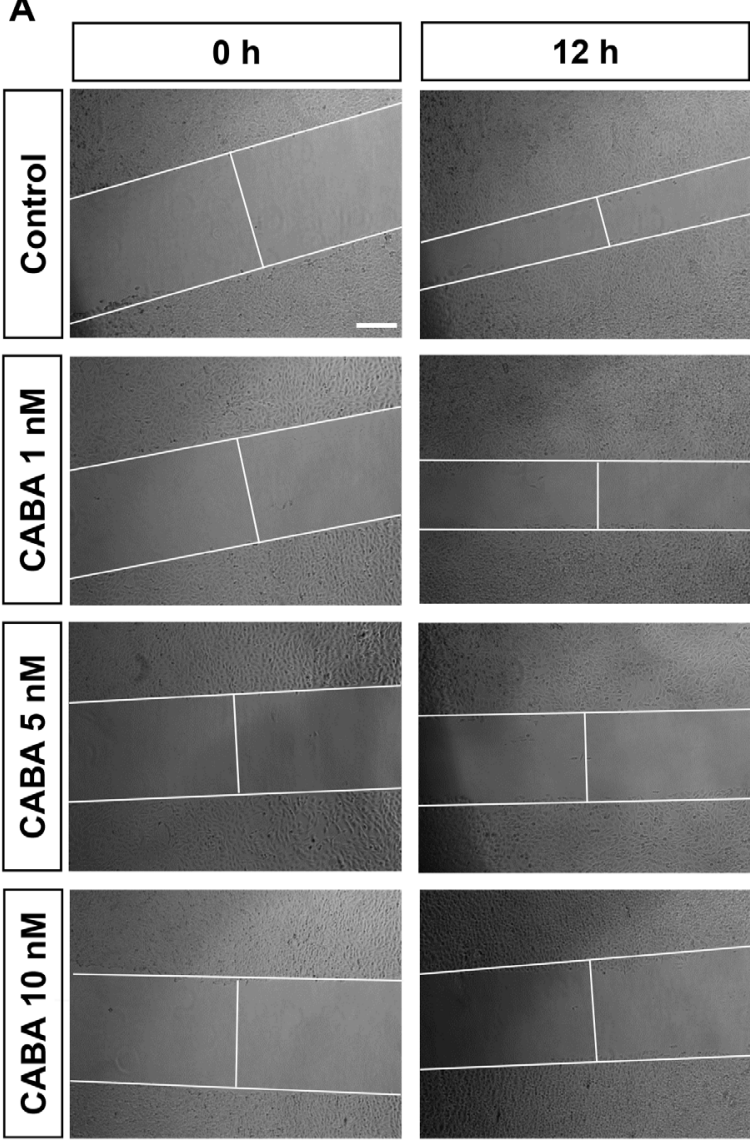

B
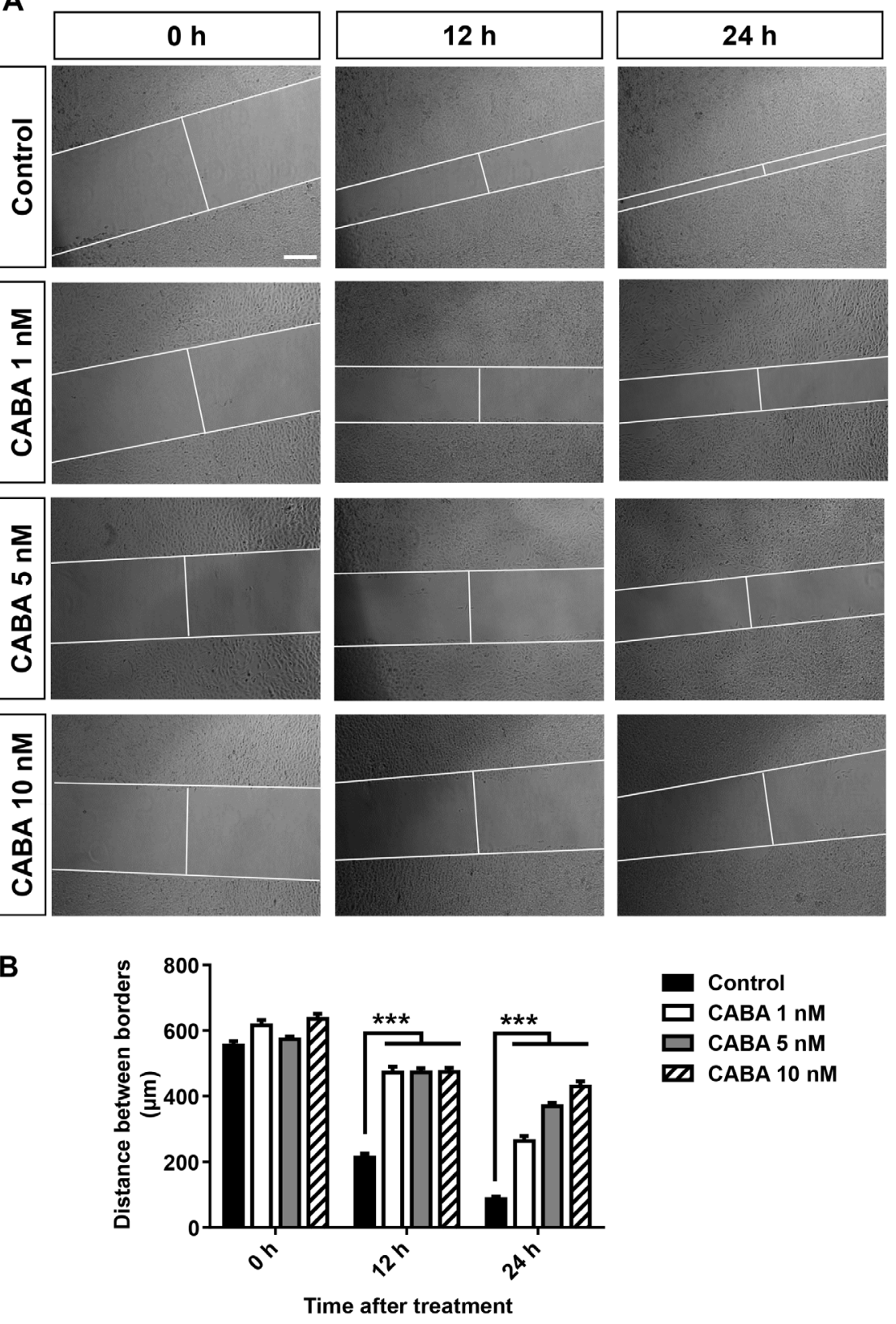

Figure 4: Cabazitaxel stalls glioma cell migration. A. The distance of scratch borders was measured 0,12 and 24 hours after treatment with various cabazitaxel concentrations $(1,5$ and $10 \mathrm{nM})$ in human glioma cells. $\mathbf{B}$. Quantification of glioma cell migration $(n=$ 3 per group). Statistical analysis was performed with One-way ANOVA (values are given as mean \pm s.e.m.). 
demonstrate that cabazitaxel is highly potent to suppress glioma motility and glioma invasion.

\section{Cabazitaxel inhibits invasive cell growth and disrupts cytoskeletal organization}

Next, we investigated the impact of cabazitaxel on glioma invasion. For this we embedded glioma cells in a 3D matrix and observed tumor cell spreading and invasion (Figure 5A, 5B). Following cabazitaxel application glioma cell spreading was significantly reduced (Figure 5B). Moreover, glioma cell morphology appeared challenged with reduced cell membrane extensions compared to untreated cells (Figure 5A). We further investigated this phenomenon and monitored the cytoskeleton following cabazitaxel application (Figure 5C). Under control conditions glioma cells showed regularly organized F-actin bundles in the cytoskeleton (Figure 5C). In contrast, glioma cells following cabazitaxel incubation showed disrupted actin fibers with G-actin like formations

\section{A}
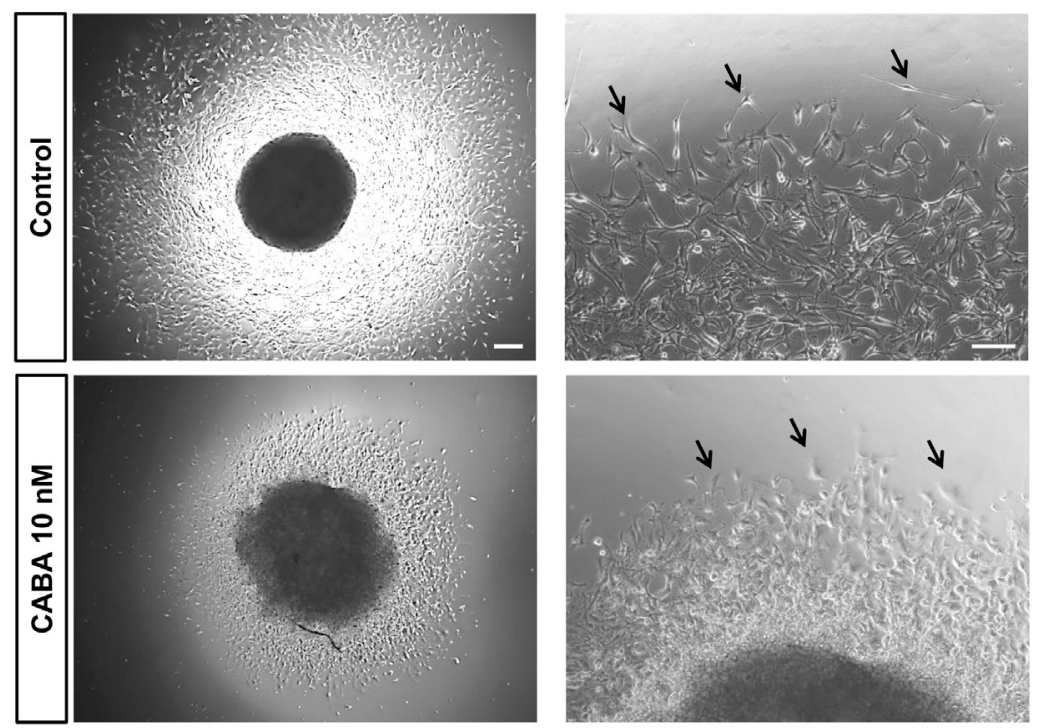

$\mathbf{B}$

C

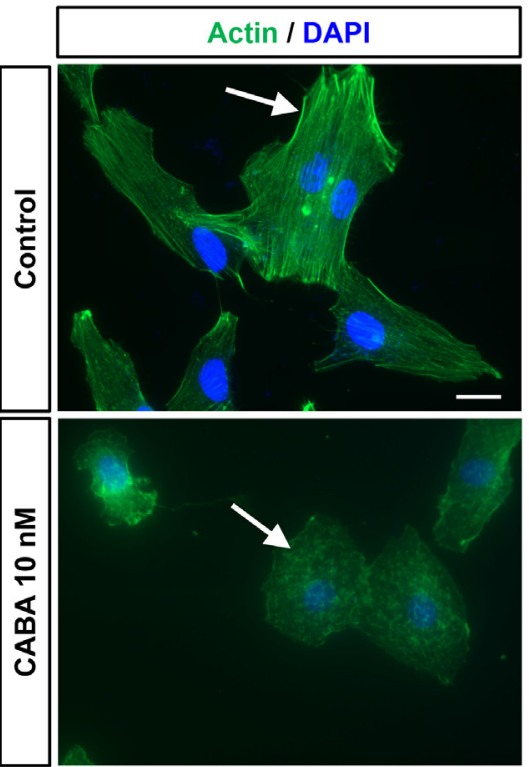

Figure 5: Cabazitaxel inhibits invasive growth and spreading of glioma cells and disrupts F-actin bundles. A. Glioma cell migration was monitored via the spheroid spreading assay. Left, representative images of human glioma cell clusters from controls and cabazitaxel (CABA) treated gliomas. Asterisks indicate glioma cell migration fronts. B. Quantification of the spheroid spreading assay. Three independent experiments were carried out and differences were considered statistically significant with values given as mean \pm SD $(n=5$; One-way ANOVA). C. Analysis of cytoskeletal F-actin distribution in human glioma cells under control conditions and following cabazitaxel treatment (CABA). Note the F-actin fiber bundles in controls, whereas following cabazitaxel treatment sole G-actin forms is detectable. Actin staining is given in green, nuclei are displayed in blue. 
in the cytoskeleton (Figure 5C). Thus, our data show that cabazitaxel acts on the actin cytoskeleton and inhibits tumor invasion.

\section{Cabazitaxel stalls tumor growth in the ex vivo VOGiM assay}

Hence, we investigated the impact of cabazitaxel in a complex tumor microenvironment. For this we acquired the ex vivo vascular glioma impact method (VOGiM) to measure tumor indexes and characteristics such as volume, death and peritumoral angiogenesis (see Material and methods). The VOGiM assay allows to study whether cabazitaxel negatively influences tumor growth in ex vivo culture. Therefore, rat brains from postnatal day four were prepared and dissected in sagittal plane. After 24 hours incubation step of brain slices, GFP positive glioma cells were implanted into the cortex. After overnight incubation, tumor implanted brain slices were treated with $5 \mathrm{nM}$ and $10 \mathrm{nM}$ cabazitaxel. One day after treatment, both $5 \mathrm{nM}$ and $10 \mathrm{nM}$ cabazitaxel (CABA) concentrations did not change tumor size compared to untreated control samples (Figure 6A). However, longer treatment durations of both cabazitaxel concentrations for four to six days significantly led to tumor growth reduction in comparison to untreated control samples (Figure 6A, 6B). After four days treatment, quantification revealed increased tumor reduction (aprox. $50 \%$ ) in cabazitaxel treated brains compared to untreated

A

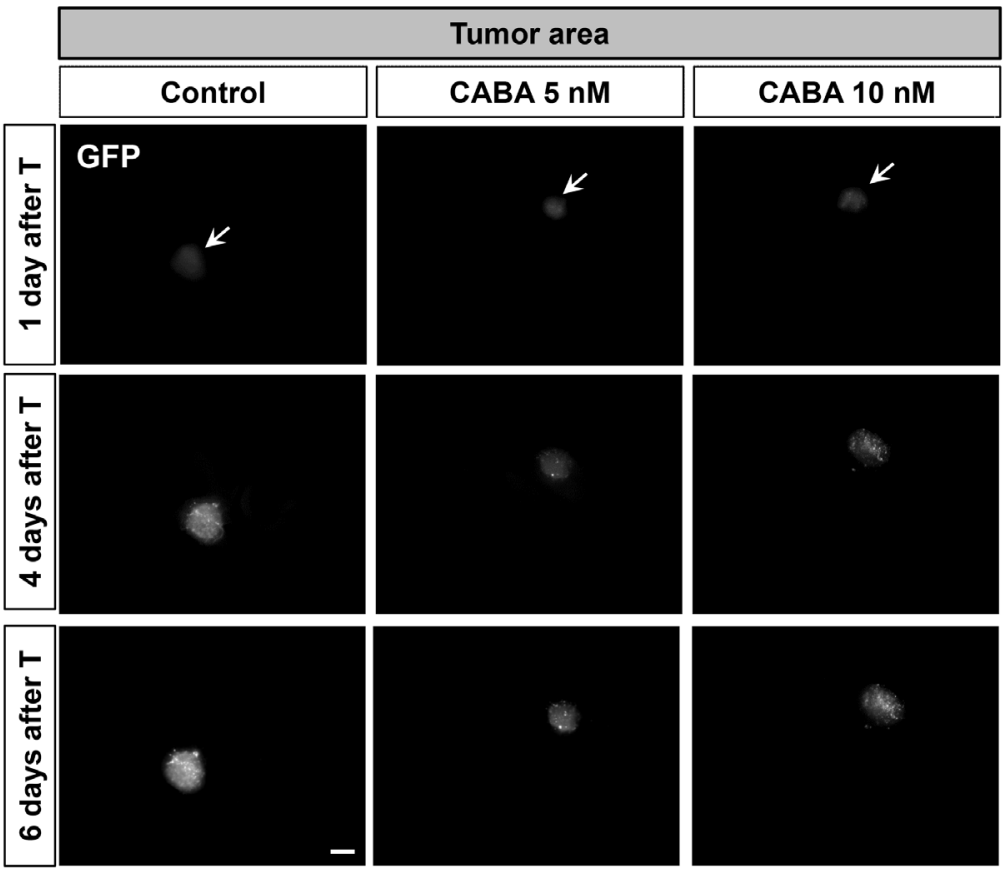

B

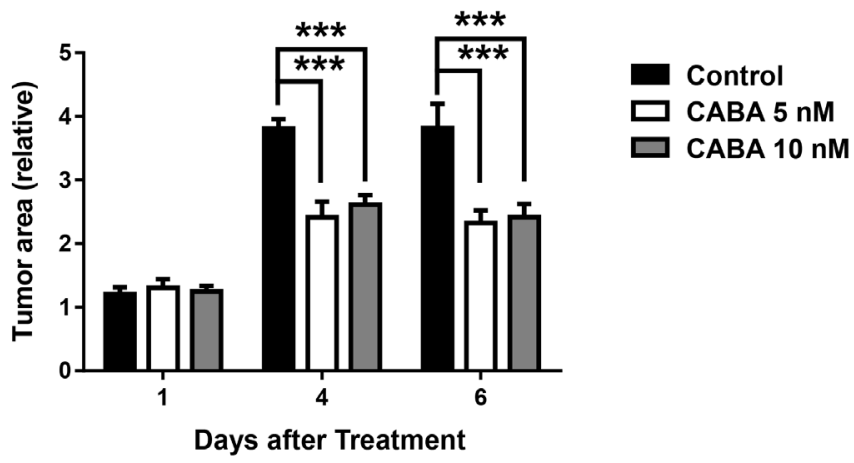

Figure 6: Cabazitaxel reduces glioma growth and tumor size in ex vivo VOGiM cultures. A. Tumor growth is illustrated in an ex vivo Organotypic Glioma Impact Model (VOGiM) as described in material and methods for 6 days after treatment. The rat brain slices were treated with 5 and $10 \mathrm{nM}$ cabazitaxel concentrations. Control samples were treated with DMSO as vehicle. B. Tumor size measurement with Image J software at 1,4 and 6 days after treatment $(n=9)$. Scale bar represents $500 \mu \mathrm{m}$. Statistical analysis was performed with One-way ANOVA $(* \mathrm{P}<0.05, * * \mathrm{P}<0.01, * * * \mathrm{P}<0.001$ and error bars represent mean \pm s.e.m. $)$. 
controls (Figure 6B). These data confirm well the potency of cabazitaxel on suppressing glioma growth rate in $e x$ vivo brain sections (Figure 6B). Noteworthy is the fact, that both $5 \mathrm{nM}$ and $10 \mathrm{nM}$ cabazitaxel were effective in inhibiting tumor growth, indicating concentrations which are reachable also in patients. Taken together, the results confirm the suppressive cabazitaxel influence on tumor growth in both in vitro and ex vivo cultures.

\section{Cabazitaxel induces tumor cell death in ex vivo VOGiM cultures}

We could show in the VOGiM assay that cabazitaxel reduced implanted tumor size in a complex tumor microenvironment. The question remained whether these tumor reducing effects of cabazitaxel were due to growth arrest or apoptosis and secondary necrosis. Therefore, we simultaneously monitored tumor cell death in the VOGiM. Cell death measurements via the marker propidium iodide (PI) demonstrated increasing cell death in the tumor area corresponding to higher cabazitaxel concentrations (Figure 7A, yellow circle). After four days of treatment with $5 \mathrm{nM}$ and $10 \mathrm{nM}$ cabazitaxel were already effective in increasing tumor cell death compared to untreated control samples (Figure 7A, 7B). However, cabazitaxel treatment with both levels resulted in up to $25 \%$ tumor cell death increase after six days (Figure 7A, 7B). Moreover, both concentrations illustrated similar cell death impact

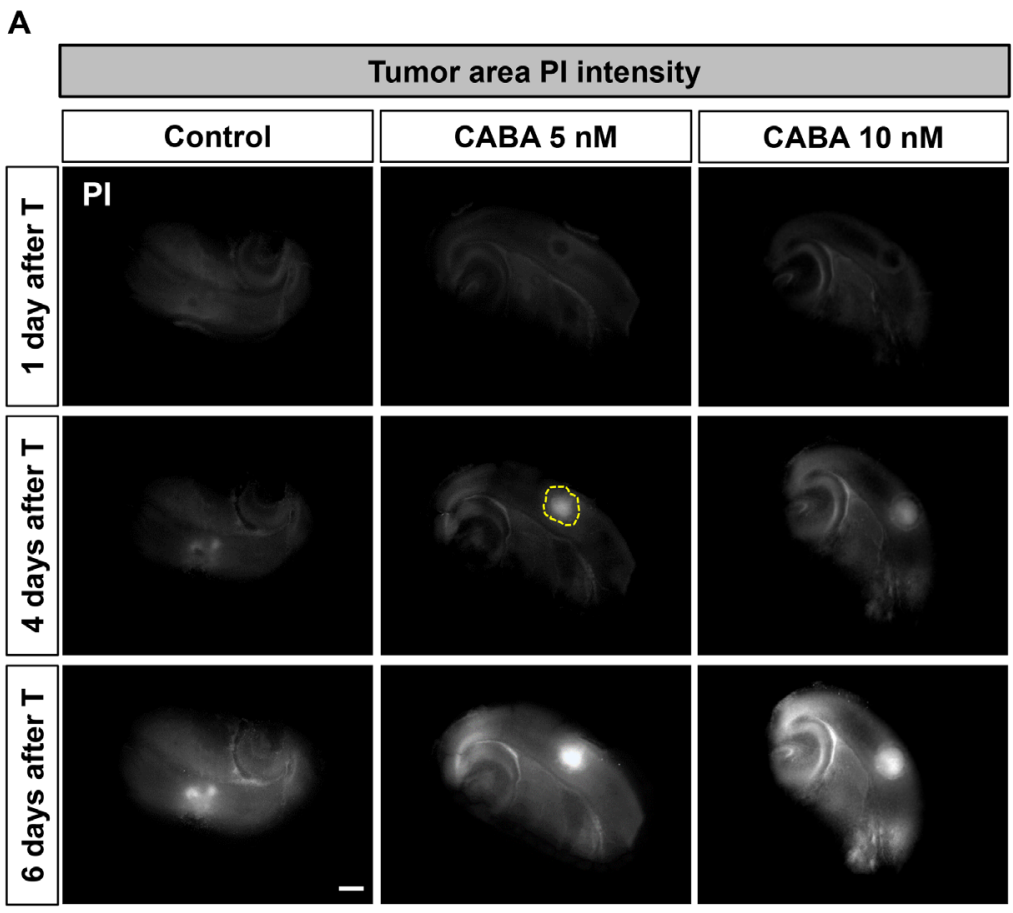

B

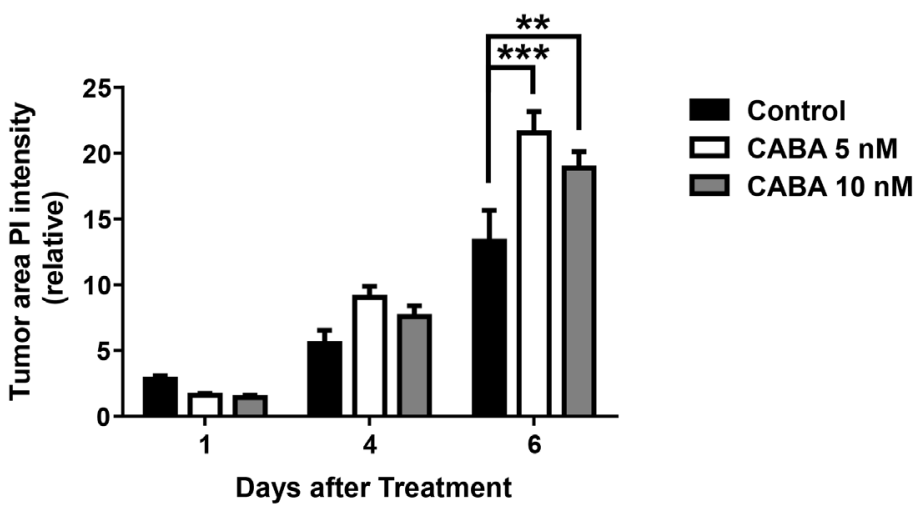

Figure 7: Cabazitaxel induces specifically tumor cell death in the brain microenviroment. A. Tumor cell death analysis which illustrated by PI intensity in the tumor area at 1,4 and 6 days after treatment. The rat brain slices were treated with $5 \mathrm{nM}$ or $10 \mathrm{nM}$ cabazitaxel. Control samples were treated with DMSO as vehicle. Yellow circle displays tumor area. B. Quantification of PI integrated intensity with Image J software at 1, 4 and 6 days after treatment $(n=9)$. Scale bar represents $500 \mu \mathrm{m}$. Statistical analysis was performed with One-way ANOVA ( $* \mathrm{P}<0.05, * * \mathrm{P}<0.01, * * * \mathrm{P}<0.001$ and error bars represent mean \pm s.e.m.). 
which confirms data on tumor size reduction following cabazitaxel application (Figure 7B). Interestingly, cabazitaxel treatment did not significantly effect on cell death of non-tumoral area (Figure 7A). Taken together we could show that cabazitaxel enables selectively cell death in the tumor area.

\section{Cabazitaxel reduces peritumoral vascularization}

The ability of a tumor to stimulate new blood vessel formation is one hallmark of malignant gliomas. The oxygen and nutrients supplied by the vasculature are crucial for tumor expansion and invasion [18, 19]. Therefore, we sought to identify whether cabazitaxel is also able to affect peritumoral vessels formation in brain tumors in the ex vivo VOGiM assay. Therefore, we implanted glioma cells into brain slices after which we treated tumor-implanted brain sections with 5 and $10 \mathrm{nM}$ cabazitaxel. After running the VOGiM for 6 days, slices were fixed and vessels stained for laminin (Figure 8A). To assess vascularization, we did analyze in particular three vascular indexes: number of junctions, number of branches and total length (see also Material and methods).

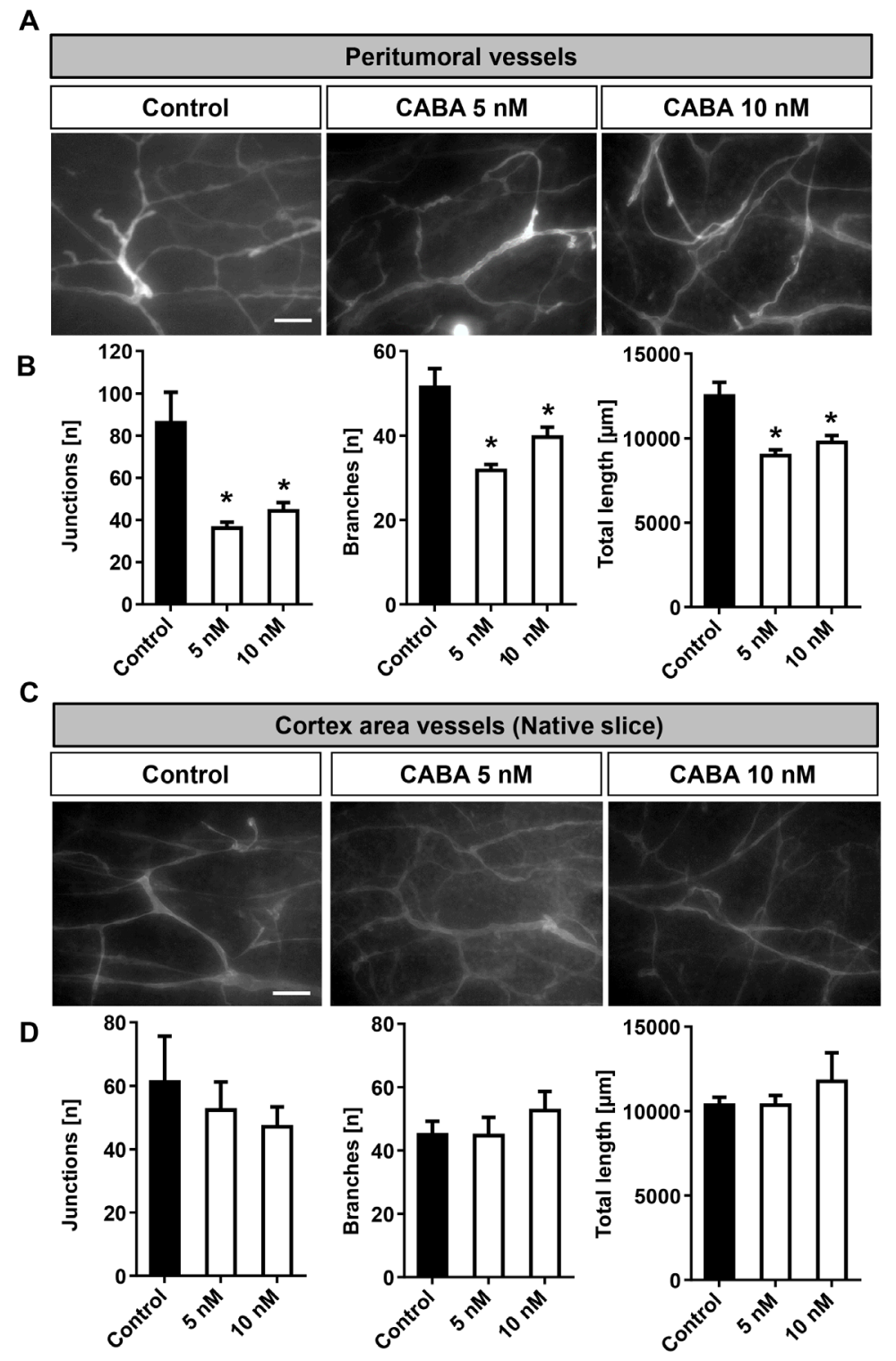

Figure 8: Cabazitaxel reduces tumor angiogenesis ex vivo. A. Laminin staining for the detection of vascularization in peritumoral area under cabazitaxel treatment. Rodent brain slices were implanted with GFP positive F98 cells and were treated with 5 and 10 nM cabazitaxel for 6 days. B. Quantification of vascularization (number of junctions, number of branches and total vessels length in peritumoral area. C. Cabazitaxel does not affect normal vessel architecture. Laminin staining for vascularization in cortex of native brain slices under 5 and $10 \mathrm{nM}$ cabazitaxel treatment. D. Quantification of physiological vascularization following cabazitaxel treatment compared to controls (number of junctions, number of branches and total vessels length in the cortex of native brain slices. Scale bar represents $50 \mu \mathrm{m}(* \mathrm{P}<0.05$, $* * \mathrm{P}<0.01, * * * \mathrm{P}<0.001$ and error bars represent mean \pm s.e.m. $)$. 
Glioma implantation induced peritumoral angiogenesis and tumoral vascularization (Figure 8A-8D). Following treatment, visualization of vessels in peritumoral area displayed clearly a reduction in vascularization in cabazitaxel treated samples (Figure $8 \mathrm{~A}, 8 \mathrm{~B})$. The quantification of vessels also demonstrated all parameters: number of junctions, number of branches and total length were decreased under both 5 and $10 \mathrm{nM}$ cabazitaxel concentrations compared to untreated control samples (Figure 8B).

Since our data revealed that cabazitaxel selectively affects the tumor microenvironment, we proceeded with analyzing the impact of cabazitaxel on normal brain vascularization. Therefore, native rat brain sections were treated with cabazitaxel for 6 days. To analyze vascularization we quantified the same vessel parameters as measured in the peritumoral area of the VOGiM setting. The results revealed that cabazitaxel did not significantly change physiological vascularization parameters (Figure $8 \mathrm{C}, 8 \mathrm{D})$. In conclusion, our results indicate that cabazitaxel is selectively impacting on peritumoral and tumor vessels whereas normal vascularization is not affected.

\section{DISCUSSION}

Malignant gliomas are intrinsic brain tumors and are the most common and lethal primary brain tumors derived from glial cells [20]. Moreover, gliomas have a high tendency to spread out into neighboring regions where they form satellite colonies [21]. In cases where neurosurgery is not applicable, chemotherapy is the first line primary treatment method for gliomas [5, 22]. However, the characteristics of tumor vascularization represent an obstacle for delivering therapeutics to tumors of the central nervous system due to challenged permeability and blood-brain barrier function $[2,23]$. The capillary endothelial cells of brain tumors are pathologically challenged in the way they lead to lack of penetration and preventing the passive diffusion of therapeutics into the brain $[10,11]$. Therefore, discovering and application of agents or small molecules with high ability to passively diffuse across the blood-brain barrier would potentiate glioma therapy. Cabazitaxel is a secondgeneration taxane which has been recently used for prostate cancer treatment $[12,16,24]$. In fact, the affinity of multidrug-resistance pumps for first generation taxanes is an obstacle narrowing the efficacy in brain tumors. However, cabazitaxel's affinity to these pumps has been shown to be rather low $[16,25]$. Moreover, cabazitaxel distributes with even pattern throughout the brain [16]. Therefore, these features make cabazitaxel a potential option for glioma first and second line treatment.

In this study, we showed that cabazitaxel is a potent cytotoxic agent for gliomas. Our results revealed that cabazitaxel treatment significantly reduced glioma survival and proliferation. Moreover, we demonstrated that low concentration of cabazitaxel was sufficiently enough to influence glioma cells. In addition, treatment of primary neurons and astrocytes with cabazitaxel did not affect cell survival or morphology. Moreover, our results also illustrated that cell survival did not change in nontumoral areas under cabazitaxel treatment in the VOGiM assay. However, concerning unintended side actions there have been published two opposing data sets. In the study of Girard and colleagues [2] the maximum tolerated dose was determined for C57BL6 mice. Mice with Smo/ Smo flank allograft tumors were treated intraperitoneal with 9,15 , or $25 \mathrm{mg} / \mathrm{kg}$ cabazitaxel for 3 days in total. Although at $25 \mathrm{mg} / \mathrm{kg}$ cabazitaxel application mice lost significantly body weight, all mice recovered from the weight loss and returned to their starting weight after 20 days [2]. In contrast, Karavelioglu and colleagues [26] reported on intraperitoneal application of cabazitaxel in Wistar rats. These authors found that cabazitaxel applied at $1.0 \mathrm{mg} / \mathrm{kg}$ body weight or higher (i.p. injection per week for consecutive 4 weeks) induced neurotoxic effects in rat brains with increased apoptosis. However, in clinical phase I trial including 21 patients with cervical, colorectal, endometrial and lung cancer received escalating dosages of cabazitaxel [31]. Eventually, a safe drug level of 25 $\mathrm{mg} / \mathrm{m}^{2}$ of cabazitaxel was recommended for use in future clinical trials.

Our data clearly show that in a setting with simultaneous presence of tumor and non-transformed cells, cabazitaxel has a higher impact on tumor cells without signs of neurotoxicity. Future studies are required to investigate in more detail the best route of cabazitaxel application and species differences in order to clarify opposing reports.

Cancer cells become accustomed to angiogenesis and for this require migratory potential as a way of guaranteeing an adequate nutrients supply [27]. In particular migration, velocity and invasive expansion are essential abilities of cancer cells allowing them to change position within compartments and tissues [28]. Our results reveal that cabazitaxel inhibits glioma cell migration and invasion. We could further show that cabazitaxel reduces tumor growth in the brain microenvironment. Tumor-implanted brain slices were treated with cabazitaxel and showed about 50\% reduction compared to control samples. Recent studies indicate that tumor microtubules are required for glioma invasion [29]. Cabazitaxel is in fact a microtubule inhibitor [30] which may explain the anti-migratory function of cabazitaxel. In addition, we found strong tumor-angiogenic inhibitory effects of cabazitaxel. This is notable since normal vascularization was not affected by Cabazitaxel. This is an important feature since the treatment of GBM is a delicate balance between aggressive elimination of cancer cells and tumor vessels and preserving brain function and protection of healthy neural tissue [1]. 
In conclusion, this report represents cabazitaxel as an efficient and selective compound to stall tumor growth and tumor angiogenesis with low cytotoxicity for brain cells. These drug features are promising for applying cabazitaxel in clinical settings.

\section{MATERIALS AND METHODS}

\section{Cell culture}

T98G human glioma cell line and U87 human glioma cell line were obtained from ATCC/LGC-2397 (Germany) and both cell lines were cultured under standard humidified conditions $\left(37^{\circ} \mathrm{C}, 5 \% \mathrm{CO} 2\right)$ with Dulbecco's Modified Eagle Medium (DMEM; Biochrom, Berlin, Germany) supplemented with 10\% fetal bovine serum (Biochrom, Berlin, Germany), 1\% Penicillin/ Streptomycin (Biochrom, Berlin, Germany) and 1\% Glutamax (Gibco/Invitrogen, California, USA). 80\% confluence glioma cells were washed with PBS for $3 \mathrm{~min}$ and then treated with trypsin $0.05 \%$ at $37^{\circ} \mathrm{C}$ for $3 \mathrm{~min}$ for dissection and then collected by gentle pipetting.

\section{Survival assay}

Cell survival was assessed by MTT assay. For this, $3 \times 10^{3}$ cells (T98G or U87 cell lines) were seeded for 3 days and incubated along with cabazitaxel (MedKoo Biosciences, USA) treatment. Cabazitaxel was added at $1,2.5,5,10,50$, and $100 \mathrm{nM}$ final concentrations. To measure survival, 3 hours before the desired time point, $10 \mu \mathrm{l}$ of MTT solution (5 $\mathrm{mg} / \mathrm{ml}$ in PBS) was added into each well and cells were incubated at $37^{\circ} \mathrm{C}$. After 3 hours incubation, the medium was removed and $100 \mu \mathrm{l}$ of isopropanol / 37\% HCL (Roche, Germany) was added into each well. The absorbance was detected at $570 \mathrm{~nm}$ with a Microplate Reader (TECAN).

\section{Apoptosis and cell cycle analysis}

Cell cycle analysis was performed according to Sehm et al. [32]. Briefly, cells were seeded on culture dishes and kept under standard conditions for 3 days. Hence, cells were isolated, washed thrice in PBS and resuspended in hypolysis buffer containing $0.1 \%$ sodium citrate, $0.1 \%$ Triton X-100, and $100 \mathrm{~g} / \mathrm{ml}$ RNAse A. Nuclei were then stained with 7AAD and analyzed by fluorescence-activated cell sorting (FACS) [32]. Cell cycle phases were analyzed with Cellsoft software.

\section{Migration assay}

To perform glioma migration, glioma cells lines were allowed to grow to $10 \%$ FBS containing DMEM medium to confluence (more than 90\%) as described previously [33]. One $\mathrm{mm}$ wide cell-free scratch wound was made across the cell layer by a sterile pipette tip. After washing twice with serum-free medium, medium was changed to new medium containing 5 and $10 \mathrm{nM}$ cabazitaxel and immediately imaged after scratching as $0 \mathrm{~h}$. Plates were photographed after 12 and 24 hours at the same location of initial image to measure glioma migration. Image J software was used for further analysis.

\section{Spheroid invasion assay}

For the spheroid assay cells were embedded in methyl-cellulose and the invasion front was quantified as described recently [33].

\section{Ex vivo VOGiM brain slice cultures and tumor implantation}

An extended description of the VOGiM assay has been presented previously [17]. Briefly, postnatal Wistar rat pups (Charles River, Boston, MA, USA) were decapitated. Brains were cautiously prepared and fixed to cut horizontally the brain into $350 \mu \mathrm{m}$ thick slices. Slices were incubated for one day in culture medium. Next day, GPF-expressing F98 cells were implanted into the brain slices. After $24 \mathrm{~h}$, brain slices were treated with 5 and $10 \mathrm{nM}$ cabazitaxel. In parallel, brain slices were also treated with propidium iodide to measure cell death. Brain slices were photographed 1, 4 and 6 days after tumor implantation to monitor tumor volume and death [17].

\section{Immunofluorescence staining}

Primary neurons and astrocytes were fixed with 4\% paraformaldehyde (Sigma-Aldrich) for $20 \mathrm{~min}$ in room temperature, washed with PBS twice followed by permeabilization for $30 \mathrm{~min}$ with $0.4 \%$ Triton-X (Sigma-Aldrich) in PBS. Primary $\beta$-tubulin III (Tuj-1) (Promega) and GFAP antibodies (Dako) were diluted in $0.4 \%$ Triton-X (Sigma-Aldrich) $+3 \%$ FCS as the blocking solution and subsequently incubated with the samples for overnight at $4^{\circ} \mathrm{C}$. Samples were washed with PBS and subsequently labeled with Alexa Fluor 488 and 568 (Invitrogen/LifeTechnologies) as secondary antibodies for $60 \mathrm{~min}$ at $37^{\circ} \mathrm{C}$. Hoechst dye (Invitrogen/ LifeTechnologies and Sigma Aldrich) was used to stain nuclei.

For vascular brain staining, brain slices were fixed with 4\% paraformaldehyde for $60 \mathrm{~min}$ in room temperature, washed with PBS twice followed by permeabilization for 30 min with $0.4 \%$ Triton-X in PBS. Then, brain slices were incubated with anti-laminin antibody which was prepared with $0.4 \%$ Triton-X + $3 \% \mathrm{FCS}$ for overnight at $4^{\circ} \mathrm{C}$. After washing with $\mathrm{PBS}$, samples were incubated with Alexa Fluor 568 for another overnight at $4^{\circ} \mathrm{C}$. Hoechst dye (Sigma Aldrich Chemicals) was used to stain nuclei. 


\section{ACKNOWLEDGMENTS}

We thank all members of the Translational Cell Biology \& Neurooncology lab for continuous experimental help and suggestions on the manuscript. This study was in part supported by Sanofi-Aventis Deutschland GmbH (\#019278).

\section{CONFLICTS OF INTEREST}

This study was in part supported by Sanofi-Aventis Deutschland GmbH (\#019278). However, the funding body had no influence on data acquisition, evaluation or presentation.

\section{Author contributions}

N.E.S. conceived and designed the study. A.G. and G.H-M. performed all experiments with help from T.S. A.G. and G.H-M. analyzed the data. A.G., I.Y.E., H.T., S.W. and N.E.S. interpreted and discussed the data with all authors. N.E.S. wrote the manuscript in conjunction with A.G. All authors contributed to the preparation of the final manuscript.

\section{Abbreviations}

CABA, Cabazitaxel; EMA, European Medicines Agency; FDA, Food and Drug Administration; GFP, green fluorescent protein; PI, propidium iodide; VOGiM, Vascular organotypic glioma impact and invasion model.

\section{REFERENCES}

1. Eyupoglu IY, Buchfelder M, Savaskan NE. Surgical resection of malignant gliomas-role in optimizing patient outcome. Nature reviews Neurology. 2013; 9:141-151.

2. Girard E, Ditzler S, Lee D, Richards A, Yagle K, Park J, Eslamy H, Bobilev D, Vrignaud P, Olson J. Efficacy of cabazitaxel in mouse models of pediatric brain tumors. Neuro-Oncology. 2015; 17:107-115.

3. Mueller S, Chang S. Pediatric Brain Tumors: Current Treatment Strategies and Future Therapeutic Approaches. Neurotherapeutics. 2009; 6:570-586.

4. Imai K, Takaoka A. Comparing antibody and smallmolecule therapies for cancer. Nat Rev Cancer. 2006; 6:714-727

5. Han B, Park D, Li R, Xie M, Owonikoko TK, Zhang G, Sica GL, Ding C, Zhou J, Magis AT, Chen ZG, Shin DM, Ramalingam SS, et al. Small-Molecule Bcl2 BH4 Antagonist for Lung Cancer Therapy. Cancer cell. 2015; 27:852-863

6. Hatipoglu G, Hock SW, Weiss R, Fan Z, Sehm T, Ghoochani A, Buchfelder M, Savaskan NE, Eyüpoglu IY.
Sunitinib impedes brain tumor progression and reduces tumor-induced neurodegeneration in the microenvironment. Cancer science. 2015; 106:160-170.

7. Brewster ME, Raghavan K, Pop E, Bodor N. Enhanced delivery of ganciclovir to the brain through the use of redox targeting. Antimicrobial agents and chemotherapy. 1994; $38: 817-823$

8. Witt KA, Gillespie TJ, Huber JD, Egleton RD, Davis TP. Peptide drug modifications to enhance bioavailability and blood-brain barrier permeability. Peptides. 2001; 22:2329-2343.

9. Willis CL. Glia-induced reversible disruption of bloodbrain barrier integrity and neuropathological response of the neurovascular unit. Toxicologic pathology. 2011; 39:172-185.

10. Furuse M, Hirase T, Itoh M, Nagafuchi A, Yonemura S, Tsukita S. Occludin: a novel integral membrane protein localizing at tight junctions. The Journal of cell biology. 1993; 123:1777-1788.

11. Kniesel U, Wolburg H. Tight junctions of the bloodbrain barrier. Cellular and molecular neurobiology. 2000; 20:57-76.

12. Tsao CK, Seng S, Oh WK, Galsky MD. Clinical development of cabazitaxel for the treatment of castrationresistant prostate cancer. Clinical Medicine Insights Oncology. 2011; 5:163-169.

13. Cisternino S, Bourasset F, Archimbaud Y, Semiond D, Sanderink G, Scherrmann JM. Nonlinear accumulation in the brain of the new taxoid TXD258 following saturation of P-glycoprotein at the blood-brain barrier in mice and rats. Br J Pharmacol. 2003; 138:1367-1375.

14. Gottesman MM, Fojo T, Bates SE. Multidrug resistance in cancer: role of ATP-dependent transporters. Nat Rev Cancer. 2002; 2:48-58.

15. Loscher W, Potschka H. Blood-brain barrier active efflux transporters: ATP-binding cassette gene family. NeuroRx: the journal of the American Society for Experimental NeuroTherapeutics. 2005; 2:86-98.

16. Semiond D, Sidhu SS, Bissery MC, Vrignaud P. Can taxanes provide benefit in patients with CNS tumors and in pediatric patients with tumors? An update on the preclinical development of cabazitaxel. Cancer chemotherapy and pharmacology. 2013; 72:515-528.

17. Ghoochani A, Yakubov E, Sehm T, Fan Z, Hock S, Buchfelder M, Eyüpoglu IY, Savaskan NE. A versatile ex vivo technique for assaying tumor angiogenesis and microglia in the brain. Oncotarget. 2015; 7:1838-1853. doi: 10.18632/ oncotarget.6550.

18. Ziyad S, Iruela-Arispe ML. Molecular mechanisms of tumor angiogenesis. Genes \& cancer. 2011; 2:1085-1096.

19. Quail DF, Joyce JA. Microenvironmental regulation of tumor progression and metastasis. Nature medicine. 2013; 19:1423-1437. 
20. Abbott NJ, Friedman A. Overview and introduction: the blood-brain barrier in health and disease. Epilepsia. 2012; 53:1-6.

21. Friedl P, Alexander S. Cancer invasion and the microenvironment: plasticity and reciprocity. Cell. 2011; 147:992-1009.

22. Wu M, Fan Y, Lv S, Xiao B, Ye M, Zhu X. Vincristine and temozolomide combined chemotherapy for the treatment of glioma: a comparison of solid lipid nanoparticles and nanostructured lipid carriers for dual drugs delivery. Drug delivery. 2015; 27:1-6.

23. Donnem T, Hu J, Ferguson M, Adighibe O, Snell C, Harris AL, Gatter KC, Pezzella F. Vessel co-option in primary human tumors and metastases: an obstacle to effective antiangiogenic treatment? Cancer medicine. 2013; 2:427-436.

24. Ecstein-Fraisse E, Su Z. Cabazitaxel--a next-generation taxane for the treatment of patients with metastatic castration-resistant prostate cancer. Gan to kagaku ryoho Cancer \& chemotherapy. 2014; 41:817-822.

25. Mizutani K, Tomoda M, Ohno Y, Hayashi H, Fujita Y, Kawakami K, Kameyama K, Kato T, Sugiyama T, Itoh Y, Ito M, Deguchi T. Effects of Cabazitaxel in Renal Cell Carcinoma Cell Lines. Anticancer research. 2015; 35:6671-6677.

26. Karavelioglu E, Gonul Y, Aksit H, Boyaci MG, Karademir M, Simsek N, Guven M, Atalay T, Rakip U. Cabazitaxel causes a dose-dependent central nervous system toxicity in rats. J Neurol Sci. 2016; 360:66-71.

27. Godlewski J, Newton HB, Chiocca EA, Lawler SE. MicroRNAs and glioblastoma; the stem cell connection. Cell Death Differ. 2010; 17:221-228.
28. Friedl P, Wolf $\mathrm{K}$. Tumour-cell invasion and migration: diversity and escape mechanisms. Nature Reviews Cancer. $2003 ; 3: 362-374$.

29. Osswald M, Jung E, Sahm F, Solecki G, Venkataramani V, Blaes J, Weil S, Horstmann H, Wiestler B, Syed M, Huang L, Ratliff M, Karimian Jazi K, et al. Brain tumour cells interconnect to a functional and resistant network. Nature. 2015; 528:93-98.

30. Villanueva C, Bazan F, Kim S, Demarchi M, Chaigneau, A. Thiery-Vuillemin, T. Nguyen, L. Cals, E. Dobi and X. Pivot. Cabazitaxel: a novel microtubule Cabazitaxel causes a dose-dependent central nervous system toxicity in rats. Drugs. 2011; 71:1251-1258.

31. Diéras V, Lortholary A, Laurence V, Delva R, Girre V, Livartowski A, Assadourian S, Semiond D, Pierga JY. Cabazitaxel in patients with advanced solid tumours: results of a Phase I and pharmacokinetic study. Eur J Cancer. 2013; 49:25-34.

32. Sehm T, Fan Z, Ghoochani A, Rauh M, Engelhorn T, Minakaki G, Dörfler A, Klucken J, Buchfelder M, Eyüpoglu IY, Savaskan NE. Sulfasalazine impacts on ferroptotic cell death and alleviates the tumor microenvironment and glioma-induced brain edema. Oncotarget. 2016; doi: 10.18632/oncotarget.8651.

33. Fan Z, Bittermann-Rummel P, Yakubov E, Chen D, Broggini T, Sehm T, Hatipoglu-Majernik G, Hock SW, Schwarz M, Engelhorn T, Doerfler A, Buchfelder M, Eyupoglu IY, Savaskan NE. PRG3 induces Ras-dependent oncogenic cooperation in gliomas. Oncotarget. 2016; doi: 10.18632/oncotarget.8592. 